CAHIERS DE

NARRATOLOGIE

\section{Cahiers de Narratologie}

Analyse et théorie narratives

$33 \mid 2018$

L'Art du roman chez Umberto Eco

\title{
Mohamed Bernoussi, Umberto Eco sémioticien et romancier
}

Marc Marti

\section{(2) OpenEdition}

Journals

Electronic version

URL: http://journals.openedition.org/narratologie/8529

DOI: $10.4000 /$ narratologie.8529

ISSN: 1765-307X

Publisher

LIRCES

\section{Electronic reference}

Marc Marti, « Mohamed Bernoussi, Umberto Eco sémioticien et romancier », Cahiers de Narratologie [Online], 33 | 2018, Online since 23 July 2018, connection on 25 September 2020. URL : http:// journals.openedition.org/narratologie/8529; DOI : https://doi.org/10.4000/narratologie.8529

This text was automatically generated on 25 September 2020

Article L.111-1 du Code de la propriété intellectuelle. 


\title{
Mohamed Bernoussi, Umberto Eco sémioticien et romancier
}

\author{
Marc Marti
}

\section{REFERENCES}

Bernoussi Mohamed, Umberto Eco sémioticien et romancier, Paris, Mimesis, 2017.

1 Connu par le grand public comme un auteur à succès et, dans le milieu universitaire, comme un sémioticien incontournable, Umberto Eco (1932-2016) est sans doute un des rares romanciers reconnus qui fasse aussi autorité dans le champ de la théorie littéraire. L'ouvrage publié par les éditions Mimesis propose de suivre pas-à-pas cette œuvre immense et variée, avec comme fil conducteur l'étroite relation entre les écrits académiques et les romans de l'illustre Italien. L'auteur, Mohamed Bernoussi, est professeur de sémiotique et de littérature à l'université de Meknès (Maroc), président de l'Association Marocaine de Sémiotique, vice-président pour l'Afrique de l'Association Internationale de Sémiotique. Il est l'auteur de nombreux articles et ouvrages sur la culture marocaine, la pensée d'Umberto Eco et les phénomènes culturels, comme Viator in tabula. Sémiotique de l'interculturel dans le récit de voyage, 2014 ; Sémiotique et société, 2015 ; Principes de la sémiotique du texte (traduction de l'italien), 2016.

2 La première partie de l'ouvrage, plus brève que les suivantes, compte vingt-et-une pages. Elle constitue un préambule à l'analyse. Intitulée «L'esthétique du Moyen Âge et l'esthétique moderne comme papier de tournesol méthodologique », elle interroge les sources médiévistes de la sémiotique d'Eco, qui commença sa recherche académique en se consacrant à la question de l'esthétique ou du beau chez Thomas d'Aquin. Mohamed Bernoussi détaille d'abord les coïncidences de cette première recherche avec les écrits postérieurs du sémioticien. Deux rapprochements sont marquants avec la scholastique et la pensée de Saint Augustin. En premier lieu, il y a une conception selon laquelle la philosophie «ne devrait pas expliquer le monde mais donner les instruments pour le 
comprendre et le transformer ». Ainsi, l'enseignement doit donner l'autonomie, en évitant deux entraves : l'excès de doutes et l'excès de certitudes. En second lieu, Eco luimême a rapproché le structuralisme de la scholastique, remarquant que tous deux avaient prétention à fonder une logique universelle, débouchant sur une science « leader » (la linguistique pour le structuralisme). Ce parallèle sous-tend L'œuvre ouverte $(1962)^{1}$, à laquelle le second chapitre est consacré. L'ouverture de l'œuvre est destinée à favoriser des "actes de liberté consciente». Le terme d'ouverture est cependant soigneusement inscrit dans une herméneutique, circonstancielle - variable en fonction des époques, comme l'art médiéval ou baroque - et intertextuelle, l'ouverture pouvant renvoyer à d'autres œuvres. On comprend d'autant mieux le refus de l'indétermination de la forme et de la liberté infinie postulées par d'autres approches qu'Eco récusera dans Les limites de l'interprétation (1991).

La seconde partie, la plus longue de l'ouvrage, compte soixante-neuf pages, tout en optant pour un déroulement chronologique, qui permet de suivre l'évolution de la pensée théorique d'Eco. "L'épisode structural » inaugure l'analyse avec La structure absente $\left(1968^{2}\right)$. La lecture détaillée de l'ouvrage permet à Mohamed Bernoussi de mettre en relief l'importance de la sémiotique de Peirce, en particulier l'interprétant, qui permet de considérer le signifié, non comme un "référent » mais comme une " convention culturelle ». Le problème du code est analysé dans la même perspective, tout comme la première réflexion d'Eco sur l'iconicité. Mais l'apport le plus important de livre du sémioticien est sans doute sa vision critique du pan-structuralisme, auquel il reproche de vouloir se transformer en ontologie.

4 Il trattato di semiotica generale (1975) n'a jamais été traduit entièrement en français. Ici, Eco réunit Peirce et Marx. L'idée est d'y démontrer que tout n'est pas signe, refusant ainsi l'ambition hégémonique que l'on prête à cette époque à la sémiotique, tout en réaffirmant cependant que certains objets participent de la sémiosis et, qu'à ce titre, ils peuvent être considérés comme des signes. L'ouvrage est dans la continuité d'une réflexion en mouvement, qui affine ses concepts et revient sur certains autres qui avaient été auparavant écartés, comme celui de référent. Son apport majeur, qui reste d'une grande actualité, est sans doute la réflexion finale sur la sémiotique de l'idéologie. Pour Eco, «la production signique est une praxis génératrice des forces sociales: les signes créent et interviennent perpétuellement sur le monde. La sémiotique elle aussi est praxis et action sur le monde, car elle nous renseigne sur la façon dont les signes et ceux qui les manipulent construisent le monde ».

5 Publié en italien en 1979 et en français en 1985, Lector in fabula est sans nul doute le «best-seller » académique d'Umberto Eco, une référence incontournable pour tout travail sur la réception de l'œuvre littéraire. Avec la distance, on finit par oublier la dimension avant-gardiste de l'essai à son époque. Le modèle proposé permet en effet de dépasser les apories du structuralisme, qui postulait que l'intervention interprétative pouvait être une sorte "d'impureté méthodologique». Comme précédemment, le recours à Peirce est essentiel, permettant, en particulier, d'insister sur la circonstance et le contexte, indispensables pour déterminer l'image du lecteur modèle. Ainsi, l'inférence devient le mode de fonctionnement du signe, en opposition à l'équivalence. L'étude proposée présente de façon claire (et pédagogique) les concepts qui découlent de cette idée centrale, et qui sont toujours utilisés dans l'analyse littéraire, en particulier «les compétences encyclopédiques du lecteur». Il indique cependant en conclusion le fait qu'Eco insiste bien plus sur la façon dont se construit l'activité 
d'interprétation modélique imposée par le texte que sur ses limites, une question qui restera en suspens jusqu'à la publication des Limites de l'interprétation (1990) ${ }^{3}$. Cet essai, qui compile des travaux antérieurs partiellement réécrits pour l'occasion, complète, comme le souligne justement Mohamed Bernoussi, L'œuvre ouverte. Il pose en effet « la nécessité d'une éthique qui veillerait sur toute démarche interprétative » tout en étant un avertissement des « dangers et ravages de quiconque joue avec le sens ». L'ouvrage reposerait aussi sur un "faux » différend avec Jacques Derrida, Eco ne réfutant pas le déconstructivisme comme méthode générale, mais simplement comme méthode d'analyse littéraire. Au final, Les limites de l'interprétation pose tout de même une question, celle de la place des interprétations « inacceptables». Mohamed Bernoussi rappelle que le risque de leur rejet est d'aboutir à une exclusion de toute hétérodoxie lectoriale. Kant et l'ornithorynque (1997) ${ }^{4}$ clôt la partie consacrée aux écrits théorique du sémioticien italien. L'analyse met en relief le caractère inabouti de l'essai, pris dans de nombreuses polémiques et dans lequel la réflexion tend parfois à se terminer par des pirouettes, moins convaincantes que l'argumentation des travaux précédents. La conclusion de la partie est intéressante. Mohamed Bernoussi y insiste sur la dimension philosophique de la sémiotique, science finalement ancienne, qui doit maintenir un dynamisme interne en se remettant « en question et [en] confront[ant] constamment ses théories et ses concepts aux phénomènes et aux réalités nouvelles ». Le fondement même de cette approche est qu'elle est « dirigée vers et par l'interprétation » comme le prouve l'apport de Saint Augustin dans les réflexions initiales d'Eco. La partie se termine sur un plaidoyer en faveur d'un usage militant de la sémiotique, auquel tout universitaire engagé dans la promotion de l'esprit critique ne peut que souscrire.

La troisième partie est consacrée à «Eco romancier ». La carrière d'écrivain, commencée sur le tard, a débuté par un best-seller planétaire, dont le succès a été renforcé par l'adaptation cinématographique de Jean-Jacques Annaud. Le succès s'explique sans doute par la double compétence d'Eco. D'une part, sa connaissance académique du Moyen-Âge, qui sert de cadre à l'action. D'autre part, sa maîtrise d'un genre littéraire de masse, le roman policier ; Le nom de la rose (1980) ${ }^{5}$ étant l'heureuse synthèse de l'ensemble. Le sacré étant au cœur de l'action, Mohamed Bernoussi souligne la représentation ambiguë de la chrétienté, qu'il rattache à la trajectoire biographique de l'auteur. Ce dernier considère le christianisme comme une culture et non comme un dogme, reconnaissant son apport à la modernité occidentale tout en critiquant son absence de remise en cause de sa vérité. Cette volonté de relativiser les vérités d'une époque, un thème qui sous-tend le roman, se retrouve en particulier dans le personnage de Guillaume, pour qui «le devoir de l'homme est de faire rire de la vérité ». Le second roman d'Eco, Le pendule de Foucault $(1988)^{6}$, fut nettement moins populaire et développait une réflexion et une fiction sur l'ensemble de la littérature ésotérique, kabbalistique, alchimique et hermétique. Par ailleurs, le texte repose sur des essais théoriques, dont Les limites de l'interprétation du propre Umberto Eco, un texte d'Adorno sur les théories du complot et une étude de Georg Simmel concernant le secret et les sociétés secrètes. Ici, le romancier s'appuie sur la réflexion du sémioticien, fabriquant une paradoxale mise en récit de la pensée hermétique, qui, théoriquement, est contraire à tout ordonnancement temporel. Avec son troisième opus, Eco renoue avec le genre historique, tout en conservant la ligne théorique, comme l'indique le titre du chapitre «La sémiotique ou la mort ». L'île du jour d'avant (1992) ${ }^{7}$ se situe au XVIIe, renvoyant à de célèbres personnages littéraires : Cervantès, Gracián, Cyrano, Pascal, Descartes... Mohamed Bernoussi considère cette œuvre comme une métaphore 
gnostique, porteuse d'une réflexion sur le temps. Baudolino $(2000)^{8}$, toujours dans le genre historique revient au Moyen Âge, sur d'une problématique assez centrale dans la réflexion sémiotique du romancier, celle du mensonge et de la fascination qu'il exerce. La mystérieuse flamme de la reine Loana (2004) ${ }^{9}$ est le roman le plus autobiographique d'Eco, une autobiographie livresque cependant. Une étude sur le rôle de l'image dans Le cimetière de Prague (2010) ${ }^{10}$ clôture l'ouvrage. L'auteur y analyse cette prédilection d'Eco pour l'insertion d'illustrations, tendance amorcée dans le roman précédent. Il pointe l'existence « de deux stratégies interprétatives et de deux instructions différentes, celle d'Umberto Eco comme auteur modèle et celle de l'auteur ou des auteurs des gravures empruntées, ce qui risque de compromettre la stratégie de départ, du moins de la parasiter ». Cette dualité est source d'ambigüité, car « comment un roman qui accorde une place de choix à des références historiques et à des illustrations qui ont valeur de documents peut-il prétendre à la littéralité ou à la fiction?». Mohamed Bernoussi propose de résoudre la polémique qu'avait suscité l'insertion de documents antisémites en revenant aux propos d'Eco, fidèle à sa ligne de romancier-sémioticien. Il révèle de fait comment le faux - historiquement support de l'antisémitisme - arrive à passer pour vrai. Les images historiques utilisées servent ainsi à déconstruire de façon critique la sémiosis antisémite telle qu'elle se forge au cours du XIXe siècle, par le faux et l'usage de faux.

7 La conclusion de l'essai renvoie finalement à ce dernier chapitre. D'une certaine façon, l'œuvre romanesque d'Umberto Eco postule un lecteur modèle, sans doute lecteur de sémiotique. Il est attendu de lui « un sens aigu de la mesure et de la critique qui lui permettront de toujours voir les signes derrières les signes, sans jamais nier totalement les choses qui avaient donné lieu à ces mêmes signes ».

\section{NOTES}

1. Version française 1965.

2. Version française en 1972.

3. Version française en 1992.

4. Version française en 1999.

5. Version française en 1982.

6. Version française en 1990.

7. Version française en 1994.

8. Version française en 2002.

9. Version française en 2005.

10. Version française en 2011. 
AUTHOR

MARC MARTI

Université Nice Côte d'Azur, LIRCES, EA3159 